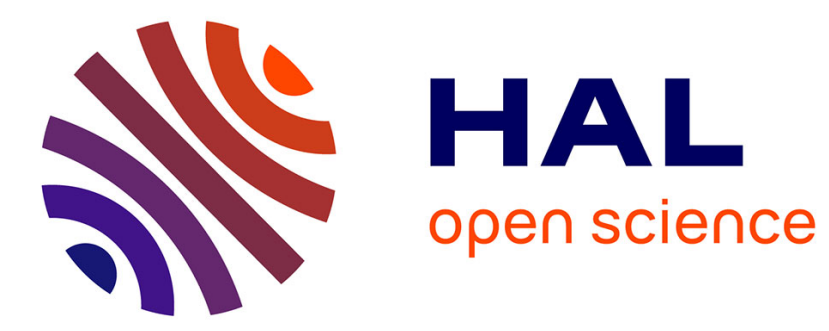

\title{
Waterfilling-based Proportional Fairness Scheduler for Downlink Non-Orthogonal Multiple Access
}

Marie Rita Hojeij, Charbel Abdel Nour, Joumana Farah, Catherine Douillard

\section{To cite this version:}

Marie Rita Hojeij, Charbel Abdel Nour, Joumana Farah, Catherine Douillard. Waterfilling-based Proportional Fairness Scheduler for Downlink Non-Orthogonal Multiple Access. IEEE Wireless Communications Letters, 2017, 6 (2), pp.230 - 233. 10.1109/LWC.2017.2665470 . hal-01511367

\section{HAL Id: hal-01511367 \\ https://hal.science/hal-01511367}

Submitted on 10 Feb 2020

HAL is a multi-disciplinary open access archive for the deposit and dissemination of scientific research documents, whether they are published or not. The documents may come from teaching and research institutions in France or abroad, or from public or private research centers.
L'archive ouverte pluridisciplinaire HAL, est destinée au dépôt et à la diffusion de documents scientifiques de niveau recherche, publiés ou non, émanant des établissements d'enseignement et de recherche français ou étrangers, des laboratoires publics ou privés. 


\title{
Waterfilling-based Proportional Fairness Scheduler for Downlink Non-Orthogonal Multiple Access
}

\author{
Marie-Rita Hojeij, Student Member, IEEE, Charbel Abdel Nour, Member, IEEE, Joumana Farah, Member, IEEE, \\ and Catherine Douillard, Senior Member, IEEE
}

\begin{abstract}
In this letter, a low-complexity waterfilling-based Power Allocation (PA) technique, incorporated within the Proportional Fairness (PF) scheduler, is proposed and applied to a NonOrthogonal Multiple Access (NOMA) scheme in a cellular downlink system. The aim of the proposed joint PA and scheduling scheme is to maximize the achieved average throughput through a quasi-optimal repartition of the transmit power among subbands, while guaranteeing a high level of fairness in resource allocation. Extensive simulation results show that the proposed technique enhances both system capacity and user fairness, when compared to either orthogonal signaling (OS) or NOMA with static PA.
\end{abstract}

Index Terms-Non-orthogonal multiple access, proportional fair scheduling, waterfilling, power allocation.

\section{INTRODUCTION}

$\mathbf{W}$ ITH the proliferation of smart and Machine-toMachine devices, it is expected that by 2021 the mobile traffic volume will be almost 10 times larger than today's [1]. To satisfy such constraints, NOMA has recently emerged as a promising candidate for future radio access. NOMA allows the cohabitation of multiple users per subband at the transmitter side, on top of the Orthogonal Frequency Division Multiplexing (OFDM) layer, and relies on Successive Interference Cancellation (SIC) [2], [3] at the receiver side.

Most of the papers dealing with NOMA consider the proportional fairness (PF) scheduler as a multiuser scheduling scheme [4], due to the good tradeoff between total user throughput and user fairness it provides. Regarding PA algorithms, equal power repartition among subbands is adopted in most cases while different multiuser PA schemes are proposed in order to distribute power among users within a subband [2], [4]. There are a few exceptions such as in [5], where a mixed combinatorial non-convex optimization problem for the maximization of the weighted sum throughput was solved using monotonic optimization, and the resulting optimal power and subcarrier allocation policy has served as a performance benchmark due to its high computational complexity. The novelty of this letter resides in the low-complexity incorporation of an inter-subband waterfilling-based PA scheme within the PF scheduler. In [2], power is maintained constant for all subbands, but an optimal PA method based on iterative

M. Hojeij, C. Abdel Nour and C. Douillard are with Institut Mines-Telecom, CNRS UMR 6285 Lab-STICC, France (email: marie.hojeij@telecom-bretagne.eu; charbel.abdelnour@telecom-bretagne.eu; catherine.douillard@telecom-bretagne.eu) and M. Hojeij is also with the Faculty of Engineering, Holy Spirit University of Kaslik, Lebanon

J. Farah is with the Faculty of Engineering, Lebanese University, Lebanon email:joumana.farah@ul.edu.lb

Part of this work has been performed in the framework of the Horizon 2020 project FANTASTIC-5G (ICT-671660), which is partly funded by the European Union. The authors would like to acknowledge the contributions of their colleagues in FANTASTIC-5G. This work has also been funded with support from the Lebanese University. waterfilling (WF) is used to allocate power among scheduled users on each subband. However, the authors state that a degraded achievable throughput occurs since the WF principle is not considered for subband allocation.

The remainder of this letter is organized as follows. We present the system description in Section II. Our proposed multiuser scheduling and PA scheme is detailed in Section III. The performance of the proposed scheme is evaluated in Section IV, and conclusions are drawn in Section V.

\section{SYSTEM DESCRIPTION}

In this letter, a downlink system with a Single Input Single Output (SISO) antenna configuration is considered. It consists of $K$ users per cell, with a total system bandwidth $B$ divided into $S$ subbands, and a maximum allowable transmit power $P_{\max }$ by the Base Station (BS). Among the $K$ users, a set of users $U_{s}=\left\{k_{1}, k_{2}, \ldots, k_{n}, \ldots, k_{n(s)}\right\}$, is selected to be scheduled over each frequency subband $s,(1 \leq s \leq S)$. $n(s)$ indicates the number of users non-orthogonally scheduled on subband $s$. A SIC process is conducted at the receiver side, and the optimum order for user decoding is in the increasing order of the users' channel gains [4] normalized by the noise and inter-cell interference $h_{s, k_{n}}^{2} / n_{s, k_{n}}$, where $h_{s, k_{n}}^{2}$ is the equivalent channel gain between user $k_{n}$ and the BS, at subband $s$, and $n_{s, k_{n}}$ the average power of the Gaussian noise plus inter-cell interference received by user $k_{n}$. Assuming successful decoding with no SIC error propagation and random inter-cell interference considered as white noise [2], the achievable throughput for user $k_{n}$, at subband $s, R_{s, k_{n}}$, is given by:

$$
R_{s, k_{n}}=\frac{B}{S} \log _{2}\left(1+\frac{h_{s, k_{n}}^{2} P_{s, k_{n}}}{\sum_{j=1, h_{s, k_{n}}^{2}<h_{s, k_{j}}^{2}}^{n(s)} h_{s, k_{n}}^{2} P_{s, k_{j}}+n_{s, k_{n}}}\right)
$$

The PA constraint is represented by the following equation, where $P_{s}$ denotes the amount of power allocated to subband $s$.

$$
\sum_{s=1}^{S} P_{s}=P_{\max }, \text { with } P_{s}=\sum_{n=1}^{n(s)} P_{s, k_{n}}
$$

\section{Multi-User Scheduling ANd Power Allocation}

The adopted scheduling policy and PA algorithm affect system efficiency and user fairness. In our previous work [6], fairness was achieved by setting user target rates, in the context of bandwidth minimization. This could be inconvenient for certain services where users are not supposed to be granted fixed data rates. Therefore, in the current work, we use 
the PF scheduler to achieve fairness. In the PF scheduler, the allocation of each subband requires the estimation of a scheduling metric for each possible user candidate (in OS) or candidate set (in NOMA). These estimations call for rate calculations which, in turn, require the power levels to be predicted on the considered subband, for each candidate. This becomes problematic as the number of subbands and/or users increases. To circumvent the power estimation problem, all previous works dealing with PF scheduling assumed equal power distribution between subbands, thus preventing intersubband power optimization. Indeed, WF, in its classical formulation, cannot be directly used within the PF, since this would necessitate performing a separate WF procedure, for each attributed subband and each candidate set, leading to a prohibitive complexity. On the contrary, in [6], setting a priority scheme allowed us to avoid the high number of tests necessary to determine the best candidate for each subband, on the one hand, and allowed the incorporation of more elaborate power allocation schemes, on the other hand. The current work aims at introducing a low-complexity iterative WF technique that allows the incorporation of the WF sub-optimal solution proposed in [6], [7] within the PF, and therefore enhance its performance.

\section{A. Proportional Fairness Scheduler}

The objective of the PF scheduler [8] is to ensure balance between cell throughput and user fairness. This scheduling policy has been adopted in the majority of papers dealing with NOMA [2], [4]. The scheduler keeps track of the average throughput $T_{k}(t)$ of each user in a past window of length $t_{c}$, where $t_{c}$ defines the throughput averaging time window (number of subframes). $T_{k}(t)$ is defined as:

$$
T_{k}(t+1)=\left(1-\frac{1}{t_{c}}\right) T_{k}(t)+\frac{1}{t_{c}} \sum_{s=1}^{S} R_{s, k}(t)
$$

where $R_{s, k}(t)$ represents the throughput of user $k$ on subband $s$, at time instance $t$. It is calculated based on (1), and can amount to zero if user $k$ is not scheduled on subband $s$.

For a subband $s$ under consideration, the PF metric is estimated for each possible users' combination $U$, and the combination that maximizes the PF metric will be denoted by $U_{s}$ :

$$
U_{s}=\underset{U}{\arg \max } \sum_{k \in U} \frac{R_{s, k}(t)}{T_{k}(t)}
$$

Since the same combinations of candidate users are tested for each subband, a user might be selected more than once and attributed multiple subbands during the same time slot. However, it can also happen that a user will not be allocated any subband. In this case, its historical rate in the following time slots will tend to be low and hence, based on the PF metric, such user will have more chance to be selected for allocation afterwards.

\section{B. Proposed Power Allocation Scheme}

We propose in this section a new low-complexity waterfilling-based PA technique that predicts the waterline level recursively from the previous level (at the allocation stage i) and from the channel gain of the considered strongest user scheduled on the current subband.

Indeed, maximizing the achieved throughput through an

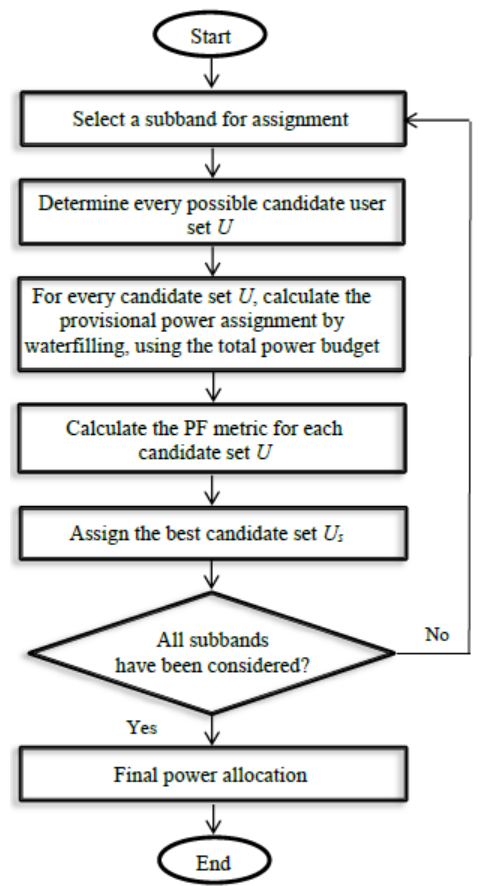

Fig. 1. Flow chart of the considered waterfilling-based allocation scheme.

optimal sharing of the total transmit power among subbands can be achieved if [9]:

$$
P_{s}+\frac{N_{0} B / S}{h_{s, k^{*}}^{2}}=W\left(S_{A}(i)\right), s \in S_{A}(i)
$$

where $S_{A}(i)$ is the set of allocated subbands at allocation stage $i, W\left(S_{A}(i)\right)$ the corresponding waterline at stage $i$, and $h_{s, k^{*}}^{2}$ the channel gain of user $k^{*}$ showing the highest channel gain among scheduled users on subband $s$.

During the allocation process, the total transmit power $P_{\max }$ is distributed, at each stage, among allocated subbands based on (5), resulting in:

$$
P_{\max }=\sum_{s \in S_{A}(i)}\left(W\left(S_{A}(i)\right)-\frac{N_{0} B / S}{h_{s, k^{*}}^{2}}\right)
$$

Since the same amount of total power is redistributed each time the scheduler allocates a new subband denoted by $s_{\text {new }}$, the waterline level is updated by $W\left(S_{A}(i+1)\right)$ only if $\frac{N_{0} B / S}{h_{s}^{2}}<W\left(S_{A}(i)\right)$, otherwise it keeps its previous value $W\left(S_{A}(i)\right)$. In case the waterline is updated, $P_{\max }$ is distributed at stage $i+1$ as follows:

$$
\begin{array}{r}
P_{\max }=\sum_{s \in S_{A}(i)}\left(W\left(S_{A}(i+1)\right)-\frac{N_{0} B / S}{h_{s, k^{*}}^{2}}\right) \\
+\left(W\left(S_{A}(i+1)\right)-\frac{N_{0} B / S}{h_{s_{\text {new }}, k^{*}}^{2}}\right)
\end{array}
$$

If we denote by $N(i)$ the number of subbands in the set $S_{A}(i)$, (6) can be re-written as:

$$
P_{\max }=N(i) \cdot W\left(S_{A}(i)\right)-\sum_{s \in S_{A}(i)} \frac{\left(N_{0} B / S\right)}{\left(h_{s, k^{*}}^{2}\right)}
$$

Hence, by comparing (7) and (8), we obtain:

$$
\begin{array}{r}
N(i) W\left(S_{A}(i)\right)-\sum_{s \in S_{A}(i)} \frac{N_{0} B / S}{h_{s, k^{*}}^{2}}=N(i) W\left(S_{A}(i+1)\right) \\
-\sum_{s \in S_{A}(i)} \frac{N_{0} B / S}{h_{s, k^{*}}^{2}}+\left(W\left(S_{A}(i+1)\right)-\frac{N_{0} B / S}{h_{s_{n e w}, k^{*}}^{2}}\right)
\end{array}
$$


Therefore, the waterline at stage $i+1$ can be formulated as:

$$
W\left(S_{A}(i+1)\right)=\frac{1}{N(i)+1}\left(N(i) \cdot W\left(S_{A}(i)\right)+\frac{N_{0} B / S}{h_{s_{n e w}, k^{*}}^{2}}\right)
$$

Fig. 1 shows the main steps of the proposed resource allocation technique that incorporates the introduced waterfilling PA within the PF.

For each new subband $s_{\text {new }}$ considered for allocation, Eq.(10) is applied for every possible candidate set of users, $U$, while taking into account user $k^{*}$ showing the highest channel gain among users in the set $U$, over $s_{\text {new }}$. Once the waterline level at the current stage, $i+1$, is determined, power is then estimated for $U$ as $P_{s_{\text {new }} \mid U}$ using:

$$
P_{s_{\text {new }} \mid U}=W\left(S_{A}(i+1)\right)-\frac{N_{0} B / S}{h_{s_{n e w}, k^{*} \mid U}^{2}}
$$

Afterwards, $P_{s_{n e w} \mid U}$ is divided among scheduled users in the set $U$ based on the chosen intra-subband PA technique, e.g. FTPA, the scheduling PF metric is calculated for each $U$ and the best set $U_{s}$ is selected based on (4). Note that, at each allocation step, the power estimation using (11) is performed only for subband $s_{\text {new }}$ in order to choose the best candidate user set, i.e., there is no need to update the provisional powers on the previously allocated subbands. The complete PA is performed only at the end of the scheduling process, after all subbands have been attributed, using the final waterline level, to yield the final power levels on all subbands.

\section{NuMERICAL RESUlts}

\section{A. System Model Parameters}

The performance of the proposed scheduling techniques are evaluated using the LTE/LTE-Advanced specifications [10]. The maximum BS transmission power is $46 \mathrm{dBm}$. The system bandwidth is $10 \mathrm{MHz}$ divided into 8, 16, 32, 64, or 128 subbands, with a carrier frequency of $2 \mathrm{GHz}$. The noise power spectral density is $4.10^{-18} \mathrm{~mW} / \mathrm{Hz}$. Users are randomly deployed in a cell of radius 500 meters, with a minimum distance of 35 meters between users and BS. Distance-dependent path loss is considered with a decay factor of 3.76. The Extended Typical Urban (ETU) channel model is used, with a mobile velocity of $50 \mathrm{~km} / \mathrm{h}$. Perfect channel estimation is assumed. Without loss of generality, in our evaluations, the maximum number of scheduled users per subband is $2(n(s)=2)$.

\section{B. Performance Evaluation}

First, two system-level performance indicators are used for evaluation: achieved system capacity and user fairness. The latter is estimated using the Gini fairness index [11] defined as:

$$
G=\frac{1}{2 K^{2} \bar{r}} \sum_{x=1}^{K} \sum_{y=1}^{K}\left|r_{x}-r_{y}\right|, \text { with } \bar{r}=\frac{1}{K} \sum_{k=1}^{K} r_{k}
$$

where $r_{k}$ is the total achieved throughput of user $k$ averaged over a time-window length $t_{c}$. $G$ takes values between 0 and 1 , where $G=0$ (resp. $G=1$ ) corresponds to the maximum (resp. lowest) level of fairness among users.

In order to evaluate our proposed PA scheme for NOMA, we compared it with an OS-based system, and with a NOMA system using EP repartition among subbands followed by an intra-subband PA based on FTPA. OS system can be regarded

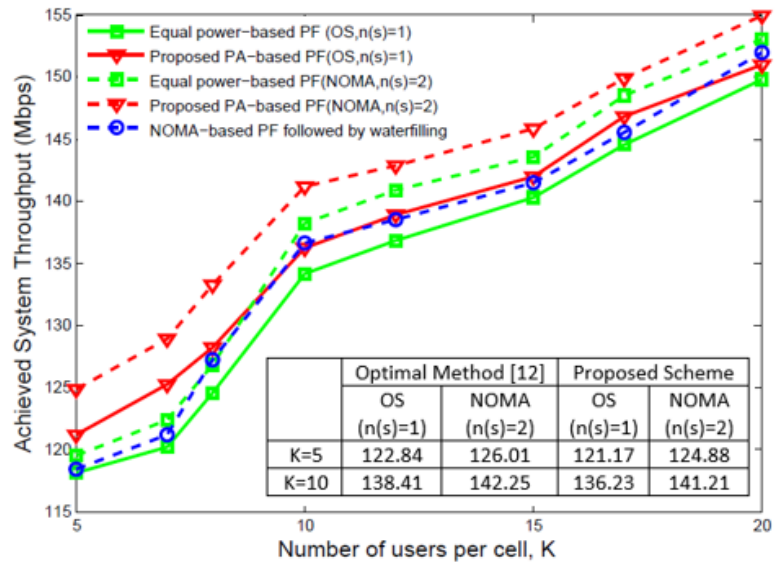

Fig. 2. Achieved system throughput in terms of $K$, for 128 subbands.

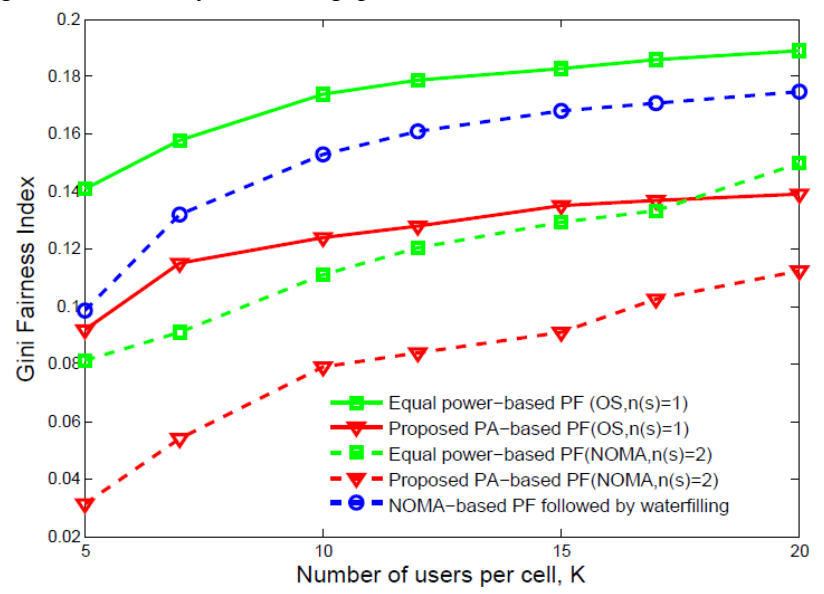

Fig. 3. Gini fairness index in terms of $K$, for 128 subbands.

as a special case of NOMA where $n(s)=1$. Note that in the NOMA case, some subbands can also be assigned to single users, leading to a hybrid scheme, such as in [4], [7].

Fig. 2 shows the achieved system throughput in terms of $K$, with a number of subbands equal to 128 . The throughput increases with the number of users per cell, for all the simulated methods. In fact, when $K$ increases, the scheduling schemes exploit the multi-user diversity more efficiently. Simulation results show that the proposed NOMA system always outperforms the OS-based system.

When compared to an equal inter-subband PA algorithm, our proposed PA scheme shows improved performance regardless of $K$, in the NOMA case as well as in the OS case. For the NOMA case, the gain in throughput can reach $5 \mathrm{Mbps}$ for 5 users per cell, i.e. $1 \mathrm{Mbps}$ per user.

A comparison of our proposed scheme with the optimal solution described in [6], and incorporated within the PF, shows that the gap between the two methods is generally $1 \%$.

Fig. 3 shows the Gini metric as a function of $K$. Fairness is significantly improved when power is dynamically distributed among subbands, independently of the access technique (OS or NOMA). However, the fairness level of NOMA is better than that of OS case. In fact, in NOMA, users having a low channel gain are given the possibility of being paired (as second users) with other users on certain subbands, and are in this case attributed a power level higher than that of the 


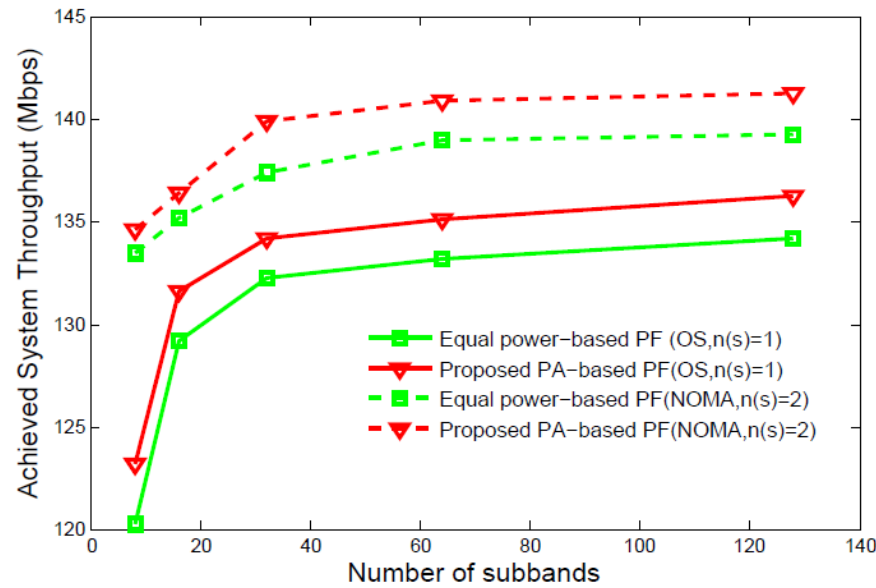

Fig. 4. Achieved system throughput in terms of $S$, for $K=10$.

users close to the center of the cell. On the contrary, when $\mathrm{PF}$ scheduling is used with OS, only one user is scheduled on each subband, therefore depriving cell-edge users from having access (as second users) to a significant number of subbands that can significantly increase their achieved data rate. From this perspective, we can see that NOMA is fairer to users than OS, since it compensates for the distance effects on the user channel quality by offering appropriate power levels.

The proposed PA scheme is also compared with an alternative method where equal inter-subband PA is considered within the PF scheduling process (to assign all subcarriers) and WF is only applied once at the end to determine the final power levels. This method (see Fig. 2 and Fig. 3) shows degraded performance with respect to the integrated WF process. This is due to the fact that users experiencing bad channel conditions but having low historical rates can be considered by the EPbased PF as having high priority on certain subbands. When applying WF at the end of the allocation, such users will be allocated a low level of power (depending on their channel gains), leading to a low spectral efficiency. The incorporation of WF within the PF allows avoiding such cases.

Fig. 4 shows the achieved system throughput as a function of $S$, for 10 users per cell. We can see that the proposed joint PA and scheduling scheme outperforms the classical NOMA $\mathrm{PF}$ even when the number of subbands is limited. As for the long-term fairness presented in Fig. 5, the gain of the proposed PA technique compared to the EP repartition is almost constant for NOMA, regardless of $S$.

From a complexity point of view, the proposed joint scheduling scheme differs from the classical PF in the waterline calculation and the power estimation step for each candidate user set. Our proposed technique increases the PF computational load by $2 S K+2 S\left(C_{K}^{2}+C_{K}^{1}\right)$ multiplications and $3 S\left(C_{K}^{2}+C_{K}^{1}\right)$ additions. As for classical NOMA PF, the calculation of the PF metric in (4) depends on the number of multiplexed users in the candidate user set. The classical NOMA PF requires a total of $3 K S+C_{K}^{1} S(4+S)+C_{K}^{2} S(13+$ $2 S)$ multiplications and $C_{K}^{1} S(1+3 S / 2)+C_{K}^{2} S(6+3 S)$ additions.

When it comes to the optimal solution [6], since it includes a numerical solver, it is not possible to compare its complexity

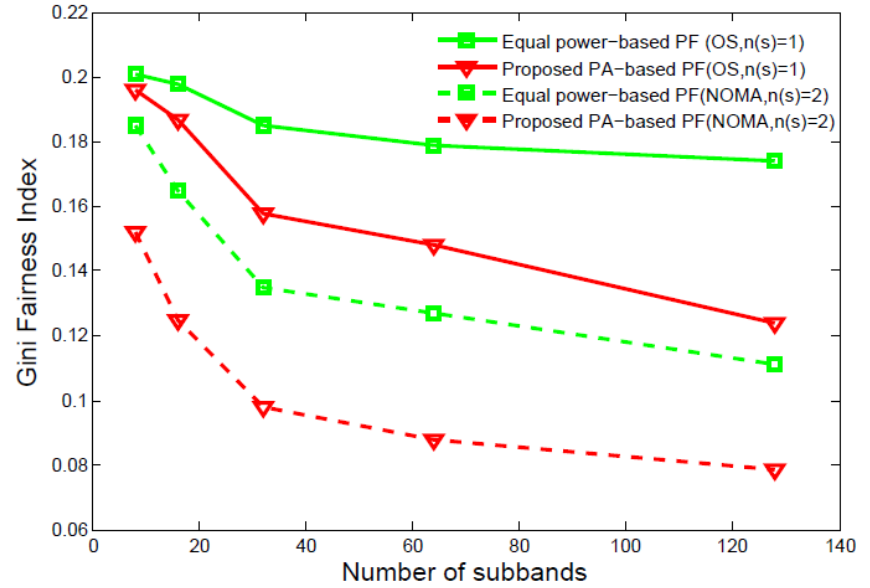

Fig. 5. Gini fairness index in terms of $S$, for $K=10$.

towards that of the suboptimal method in terms of the number of additions and multiplications. Instead, a measure of the average execution time of one complete allocation cycle (i.e. in one timeslot), for the case of 10 users per cell and 8 subbands, yielded $182745 \mathrm{~ms}$ for the optimal solution and $68 \mathrm{~ms}$ for the proposed suboptimal scheme.

\section{CONCLUSION}

In this letter, we have proposed a low-complexity waterfilling-based PF scheduling scheme. Simulation results show that the proposed scheme allows an increase in the total user throughput and in the system fairness, when compared to an OS-based system and to a NOMA system considering an equal power repartition among subbands. The study conducted here with two scheduled users per subband can be easily adapted to a larger number of paired users.

\section{REFERENCES}

[1] https://www.ericsson.com/res/docs/2016/ericsson-mobility-report2016.pdf

[2] N. Otao, Y. Kishiyama, and K. Higuchi, "Performance of non-orthogonal access with SIC in cellular downlink using proportional fair-based resource allocation", in proc. Int. Symp. on Wireless Commun. Syst.,2012, 476-480.

[3] Z. Ding, Z. Yang, P. Fan, and H. Poor, "On the performance of nonorthogonal multiple access in $5 \mathrm{G}$ systems with randomly deployed users", IEEE Signal Process. Lett., 2014, 21(12), 1501-1505.

[4] Y. Saito, A. Benjebbour, Y. Kishiyama, and T. Nakamura, "System-level performance evaluation of downlink non-orthogonal multiple access (NOMA)", in proc. IEEE PIMRC, Sept. 2013.

[5] Sun, Yan, et al. "Optimal Joint Power and Subcarrier Allocation for FullDuplex Multicarrier Non-Orthogonal Multiple Access Systems.” arXiv preprint arXiv:1607.02668 (2016).

[6] M.R. Hojeij, J. Farah, C. Abdel Nour, and C. Douillard, "New Optimal and Suboptimal Resource Allocation Techniques for Downlink Nonorthogonal Multiple Access", Wireless Pers. Commun., 2015, 1-31.

[7] M.R. Hojeij, J. Farah, C. AbdelNour, and C. Douillard, "Resource Allocation in Downlink Non-Orthogonal Multiple Access (NOMA) for Future Radio Access", in proc. 81st IEEE VTC, 2015.

[8] F.P. Kelly, a.K. Maulloo, and D.K.H. Tan, "Rate control for communication networks: shadow prices, proportional fairness and stability"', $J$. of the Operational Research Soc, 1998, 49(3), 237-252.

[9] S. Haykin, Communications Systems, 4th ed. Colorado: Wiley, ch.6, pp. 451-460.

[10] 3GPP, TR25-814 (V7.1.0), "Physical Layer Aspects for Evolved UTRA", 2006.

[11] M. Dianati, X. Shen, and S. Naik, "A new fairness index for radio resource allocation in wireless networks", in Proc. IEEE Wireless Commun. and Netw. Conf., 2005. 